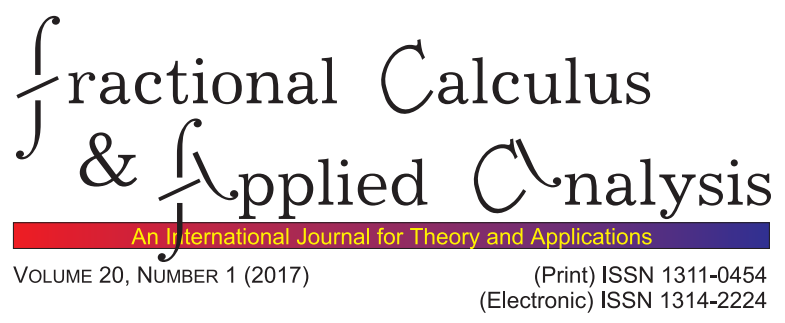

\title{
EDITORIAL
}

\section{FCAA RELATED NEWS, EVENTS AND BOOKS}

(FCAA-VOLUME 20-1-2017)

\section{Virginia Kiryakova}

Dear readers,

in the Editorial Notes we announce news for our journal, anniversaries, information on international meetings, events, new books, etc. related to the FCAA ("Fractional Calculus and Applied Analysis") areas.

\section{Calendar of FC Related Events}

For other FC related events in 2017, see previous FCAA Editorial Notes in Vol. 19, No 4, 5, 6 (2016). All of them are available online free at journal's website https://www.degruyter.com/view/j/fca, select volume and issue.

7th International Scientific Conference, OTHA 2017, "Modern Methods, Problems and Applications of Operator

Theory and Harmonic Analysis VII"

(Rostov-na-Don, Russia, April 23-28, 2017)

More details can be found on

Website: http://otha.sfedu.ru/conf2017/.

The conference is dedicated to the 75 anniversary of Professor Nikolai K. Karapetiants (1942-2005).

Working days of the conference: 24-27 of April 2017; Arrival: April, 23rd; Departure: April, 28th. Deadline for registration and for abstracts submission: 01 April, 2017.

Sessions: - Functional Analysis and Operator Theory; - Function Theory and Approximation Theory; - Differential Equations and Mathematical Physics (Chair - Vladislav V. Kravchenko); - Hausdorff Operators and Related Topics (Chair - Elijah Liflyand); - Probability-Analytical Models and Methods (Chair - Igor V. Pavlov); - Bioinformatics and Mathematical Modelling (Chair - Alexander V. Melerzanov).

For contacts, E-mail: otha.conference@gmail.com (Alexey Gil).

We look forward to receiving your contribution. Yours sincerely, Alexey N. Karapetyants

(c) 2016 Diogenes Co., Sofia

pp. 1-6, DOI: 10.1515/fca-2017-0001

DE GRUYTER 


\section{Workshop on Mathematical Methods in Engineering, MME 2017 \\ (Ankara, Turkey, April 27-29, 2017)}

It is organized by organized by Cankaya University.

More details can be found on

Website: http://mme2017.cankaya.edu.tr/.

Main Topics:

- Emergent Mathematics-Supported Data Mining and Prediction Tools; - Dynamics of Complex Systems; - Fixed Point Theory and Applications; - Fractals; - Fractional Calculus and Applications; - Fuzzy Sets and Systems; - Image and Signal Analysis; - Mechatronics; - Nonlinear Dynamics; - Ordinary Differential Equations and Applications; - Partial Differential Equations and Applications; - Planning and Scheduling Modelling; - Quantum calculus and its applications; - Stochastic Hybrid Systems; - Stochastic Optimal Control; - Vibration and Control.

Important Dates: - Deadline for draft papers submission: February 15, 2017; - Notification of acceptance: March 15, 2017; - Final manuscript and registration: April 10, 2017.

Proceedings of the workshop are planned as:

- Special issue in Advances in Difference Equations (Guest Editors: K. Tas and D. Baleanu);

- Special issue entitled "New Trends in Fractional Modelling of Transport Problems in Fluid Mechanics and Heat-Mass Transfer", in Journal Thermal Science;

- CD with ISBN number; - Edited Springer book.

Yours sincerely, Prof. Dr. Kenan Tas, Chair of MME-2017

Contact info, E-mail: mme2017@cankaya.edu.tr

Communicated by: J. Tenreiro Machado (Co-Chair)

\section{3rd edition of ECCTD 2017}

\section{European Conference on Circuit Theory and Design 2017}

(Catania, Italy, September 4-6, 2017)

It is organized by organized by the DIEEI (Electric, Electronics and Computer Engineering Department) of the University of Catania.

More details can be found on

Website: http://www.ecctd2017.dieei.unict.it, also at: http://conference.researchbib.com/view/event/64795 
Topics: - Circuits; -Systems; - Mathematical and computational methods; - Computational methods; - Nanoscale devices \& circuits; - Neuromorphic \& biomedical circuits; - Control of complex networks; - Signal processing applications.

Special session is planned at ECCTD 2017, the topic is:

"Progress on fractional-order devices and systems in interdisciplinary applications", organized by:

- Riccardo Caponetto (Italy): riccardo.caponetto@dieei.unict.it, - Ahmed S. Elwakil (UAE): elwakil@ieee.org, - Costas Psychalinos (Greece): cpsychal@physics.upatras.gr, - Ivo Petras (Slovak Republic): ivo.petras@tuke.sk.

Major headlines of the special session: - Synthesis and design of fractional-order circuits and systems; - Modeling and identification applications of fractional-order systems; - Novel fractional-order control techniques; Applications of fractional-order circuits in biology, biomedicine, renewable energy etc.; - Fractional-order devices and their applications.

Colleagues interested in this special session are invited to send to its organizers a title and abstract of proposed contribution, before February 15, 2017 or ASAP.

Important dates for the conference: - Special session proposals: 15.02.17; - Notification of acceptance of special sessions: 01.03.17; - Paper submission deadline (4 pages): 07.04.17; - Notification of paper acceptance: 30.05.17.

Call for papers, at: http://www.ecctd2017.dieei.unict.it/CallforPapersECCTD2017.pdf.

The conference will be followed by the 12th SICC International Tutorial Workshop "Topics in nonlinear dynamics" on the topic of Control of Complex Networks of Nonlinear Circuits and Systems, September 7-8, 2017, more details at http://www.ecctd2017.dieei.unict.it/SICCWorkshop.html.

Contacts, General Chair, E-mail: ecctd2017@unict.it (Mattia Frasca) Communicated by: Ivo Petras and Riccardo Caponetto

\section{International Conference on Fractional Signals and Systems, FSS 2017 \\ (Lodz, Poland, September 9-11, 2017)}

It is organized by organized by Institute of Applied Computer Science, Lodz University of Technology.

More details can be found on

Website: http://www.fss17.p.lodz.pl/. 
EDITORIAL

The conference will offer three stimulating days of newest results presentation and discussions. It encompasses a broad spectrum of the Fractional Calculus applications in technical sciences. The main tracks will be: the fractional-order continuous-, and discrete-time linear or non-linear fractional-order control, dynamic system identification via fractional models, fractional order filtering, image processing using fractional methods. The mentioned range is not meant to exclude other applicable areas.

Main topics: - Fractional order control; - Signal analysis and filtering with fractional tools; - Fractional modeling; - Fractional system identification; - Image processing using methods based on the fractional calculus; Numerical methods for fractional calculus.

Important dates: - Early registration deadline: March 1st, 2017; Mini-symposiums proposal: March 15th, 2017; - Submission deadline: May 15th, 2017; - Notification of acceptance: 15th June, 2017; - Submission of final version: 1st July, 2017.

Contacts: fss17@info.p.lodz.pl

Communicated by: Piotr Ostalczyk

\section{2nd IEEE International Conference ETFA 2017, Emerging Technologies for Factory Automation 2017 (Limassol, Cyprus, September 12-15, 2017)}

Details on the conference can be found on

Website: http://etfa2017.org/.

Special session is planned:

"Challenges for control structures under new automation architecture: Requirements, adaptation and auto-tuning", organized by: Clara Ionescu (Belgium), Claramihaela.ionescu@ugent.be, Margret Bauer (South Africa), margret.bauer@wits.ac.za

Author's schedule: - Proposals for special sessions: due February 12, 2017; - Special session papers: due March 12, 2017; - Notification of acceptance of special session papers: May 15, 2017; - Deadline for submission of final manuscripts: July 2, 2017.

Selected papers from ETFA2017 will be considered for publication in special sections in the IEEE Transaction on Industrial Informatics.

Further information and contacts: contact@etfa2017.org

Communicated by: Dana Copot, dana.copot@ugent.be 


\section{New Books}

J. Tenreiro Machado, António M. Lopes, Alexandra M. Galhano, Duarte Valério, Solved Problems in Dynamical Systems and Control. The Institute of Engineering and Technology - Digital Library, UK (2016), 350 pp., ISBN: 9781785611742 , e-ISBN: 9781785611759; Book DOI: 10.1049/PBCE107E, Chapter DOI: 10.1049/PBCE107E.

Details: http://digital-library.theiet.org/content/books/ce/pbce107e.

About this book: This book presents a collection of exercises on dynamical systems, modelling and control. Each topic covered includes a summary of the theoretical background, problems with solutions, and further exercises. Topics covered include: block diagram algebra and system transfer functions; mathematical models; analysis of continuous systems in the time domain; root locus analysis; frequency domain analysis; PID controller synthesis; state space analysis of continuous systems; controller synthesis by pole placement; discrete time systems and the $\mathrm{z}$ transform; analysis of non-linear systems with the describing function method; analysis of nonlinear systems with the phase plane method; and fractional order systems and controllers. Based on tried-and-tested problems and solutions that the authors use in teaching over 500 students each year, this book is essential reading for advanced students with courses in modelling and control in engineering, applied mathematics, biomathematics and physics.

Contents: 1. Block diagram algebra and system transfer functions; 2. Mathematical models; 3. Analysis of continuous systems in the time domain; 4. Root-locus analysis; 5. Frequency domain analysis; 6. PID controller synthesis; 7 . State space analysis of continuous systems; 8. Controller synthesis by pole placement; 9 . Discrete-time systems and $Z$ transform; 10. Analysis of nonlinear systems with the describing function method; 11. Analysis of nonlinear systems with the phase plane method; 12. Fractional order systems and controllers (pp. 341-363):

Derivatives and integrals can be extended to orders which are not integer. These can be used in differential equations to describe the dynamics of a system, or of a controller, in a more supple manner than with integer derivatives and integrals only.

Appendix A (pp. 365-377):

Presents a collection of tables covering the following aspects: Laplace transforms; Bode diagrams; transfer functions; Nichols plots; $Z$-transforms; nonlinearities; Grünwald-Letnikoff definitions; Riemann-Liouville definitions; and Caputo definition. 


\section{Important warning: Incorrect Information on New Books}

Dear respected readers of the FCAA journal,

I would like to inform you that somebody abused my name and published two books with my name as "author" and "co-author", respectively. This was without my knowledge and I have never given agreement to anybody to use my text, figures, tables or SW code in these books:

Author / Contributor: Ivo Petras, Advances in Numerical Methods in Engineering with MATLAB. Publisher: Auris Reference, ISBN: 9781781548257, Publ. Date: 2016;

Author / Contributor: Rekha R. Rao, Ivo Petras, Tomasz Kopecki, Encyclopaedia of Numerical methods in Engineering (3 Volumes). Publisher: Auris Reference, ISBN: 9781781548233, Publ. Date: 2016.

I do not have information what is inside these books. Please be careful if you decide to buy them.

I myself observed the information on these "books" in advertisements by Amazon.com ! I found the contact information and tried to contact the publisher's representative, but I did not receive any reply. I contacted Dr. Tomasz Kopecki who is listed as "co-author" on the same book and - in his own words - he was "surprised and shocked". I am deeply concerned with the thought that if this happened to me, then something similar can happen to anybody else.

Sincerely yours, Ivo Petras, Technical University of Kosice, Slovakia

Virginia Kiryakova, Institute of Mathematics and Informatics Bulgarian Academy of Sciences, Acad. G. Bontchev Str., Block 8 Sofia1113-BULGARIA, e-mail: virginia@diogenes.bg

Please cite to this paper as "Ed. Note, FCAA-Volume 20-1-2017", publ. in: Fract. Calc. Appl. Anal., Vol. 20, No 1 (2017), pp. 1-6, DOI: $10.1515 /$ fca-2017-0001 